\title{
Thermophilic Campylobacter spp. occurrence on chickens at farm, slaughter house and retail
}

\begin{abstract}
The aim of this study was to investigate the occurrence of campylobacters in chicken at farms (close-house system and open-house system), slaughtering (conventional slaughterhouse and processing plant) and retail (wet market and supermarket). Campylobacter spp. was not found in cloacal swabs in chickens aged of 4 weeks in farms with close-house system. Campylobacter spp. was found in cloacal swabs (95.0\%) in four weeks old chicken in farms with open-house systems. End-slaughtering samples from conventional slaughterhouse and processing plant were contaminated with Campylobacter spp. at $84.0 \%$ and $94.0 \%$, respectively. Campylobacter contamination on wet market and supermarket samples with $78.0 \%$ and $92.0 \%$, respectively. Close-house system at farm level was able to prevent or delay Campylobacter spp. colonization in chickens but contamination by Campylobacter spp. at retail level was still high. Therefore, monitoring of Campylobacter spp. in chicken products at retail level is crucial to reduce risk of human ingestion of Campylobacter spp. through chicken products.
\end{abstract}

Keyword: Campylobacter spp., farm, slaughter house, retail 